\title{
A dental anxiety scale for children
}

Development of a computerised dental anxiety scale for children: validation and reliability

\section{H. Buchanan Br Dent J 2005; 199: 359-362}

\section{Objective}

To provide reliability and validity data on a computerised dental anxiety scale (the Smiley Faces Program or SFP) for children using faces as a response set.

\section{Setting}

Five schools across Derby, Newcastle-upon-Tyne and Shropshire. Subjects and method

The SFP was completed by 464 children (mean age 10.8 years). For validity purposes, 241 children also completed two other children's dental anxiety measures (the Modified Child Dental Anxiety Scale and the Dental Fear Survey). For test-retest purposes 100 of the children completed the SFP two weeks later.

\section{Results}

The SFP demonstrated good internal consistency $(\alpha=0.8)$, test-retest reliability was good and significant correlations were found between the SFP and the other measures. The children rated the local anaesthetic and drill as the most anxiety-provoking items.

Conclusion

This study suggests that the SFP is a valid and reliable measure for assessing children's trait dental anxiety and may help encourage dentists to formally assess dental anxiety.

\section{IN BRIEF}

- Describes a computerised dental anxiety scale for children called the Smiley Faces Program (SFP).

- The SFP proved to be a reliable and valid measure for children from six years old.

- The program has the added advantage of a computer database with anxiety scores for immediate investigator access.

\section{COMMENT}

The need to be able to assess and evaluate dental anxiety has been recognised as central to establishing a treatment alliance with the adult and child patient. ${ }^{1}$ While it may be possible to engage the anxious adult patient, the same may not be so for the anxious child. The dentist's relationship with the adult patient is on a oneto-one basis whereas the relationship with the child is by necessity triangular, as it includes the accompanying adult. ${ }^{2}$ It is therefore important that a system of assessment of child dental anxiety is one which is child-centred, quick and easy to use as well as reliable and valid. Buchanan has provided the reader with just that in the Smiley Faces Program (SFP).

The SFP is a four-item computerised dental anxiety scale. The faces describe the child's response to a range of dental stimuli ranging from going to the dentist to having an injection. The questions appear on the computer screen for a matter of seconds and then the child is asked to replace the neutral face with one of seven faces which describes how they feel about the dental item.

Buchanan provides the reader with statistics to demonstrate the validity and reliability of SFP and comparisons with other child dental anxiety inventories allows for a measure of validity to be made. She points to the importance of the individual items suggesting that the SFP allows the dental health professional to assess children's fears of eg the injection. While she is cautious about the generalisability of the SFP and its use for younger children or those with learning difficulties, I feel that these fears may be unfounded - as Buchanan states: 'children with learning difficulties, or those with English as a second language may struggle with the instructions ... this would also be the case for established paper and pencil measures'. In such situations the child could be assisted to complete the SFP thus allowing a rapport between the child and health professional to be developed.

The SFP has the psychometric properties as well as the potential to engage dentally anxious children in a novel and innovative way while assessing their dental anxiety. Can there be a better way of developing rapport and the treatment alliance with the anxious child than providing a computerised system to assess their dental fear? I think not.

R. Freeman, Professor of Dental Public Health, Queen's University, Belfast

doi: 10.1038/sj.bdj.4812695

1. Burke FJ T, Freeman R. Preparing for dental practice. Oxford: Oxford University Press, 2004.

2. Freeman $R$. The case for the mother in the surgery. Br Dent J 1999; 186: 310-316 